\title{
Quasimodular forms and Poincaré series
}

by

\author{
Min Ho LeE (Cedar Falls, IA)
}

1. Introduction. Quasimodular forms were introduced by Kaneko and Zagier in [5] and have been studied actively since then in connection with various topics in number theory (see e.g. [1], [7], [9], [11]). They are also linked to some problems in applied mathematics (cf. [4], [10]). Quasimodular forms generalize modular forms, and one of their useful properties is that their derivatives are also quasimodular forms. In particular, the derivatives of modular forms are quasimodular forms. On the other hand, as shown in this paper, each quasimodular form can be expressed in terms of derivatives of some modular forms. In fact, it turns out that a quasimodular form can be identified with a finite sequence of modular forms. This identification may allow us to investigate certain aspects of quasimodular forms by studying the corresponding sequences of modular forms.

Poincaré series provide examples of classical modular forms, and one of the goals of this paper is to construct Poincaré series for quasimodular forms by using an automorphism of the space of polynomials that is equivariant under certain actions of $\mathrm{SL}(2, \mathbb{R})$. For this purpose we need to introduce two types of actions of $\mathrm{SL}(2, \mathbb{R})$ on the space of polynomials over the ring of holomorphic functions on the Poincaré upper half-plane as well as an equivariant automorphism. Given a discrete subgroup $\Gamma$ of $\mathrm{SL}(2, \mathbb{R})$, quasimodular polynomials and modular polynomials for $\Gamma$ are invariant polynomials under such actions restricted to $\Gamma$. Thus the equivariance property shows that the above automorphism induces an isomorphism between the space of quasimodular polynomials and that of modular polynomials.

The coefficients of modular polynomials are modular forms of certain weights, so that a modular polynomial can be identified with a certain finite sequence of modular forms. On the other hand, quasimodular polynomials correspond to quasimodular forms. Indeed, given integers $m$ and $w$ with $m \geq 0$, a quasimodular form $f$ of weight $w$ and depth at most $m$ for a discrete 
subgroup $\Gamma$ of $\operatorname{SL}(2, \mathbb{R})$ corresponds to holomorphic functions $f_{0}, f_{1}, \ldots, f_{m}$ on the Poincaré upper half-plane $\mathcal{H}$ in such a way that

$$
\begin{aligned}
\frac{1}{(c z+d)^{w}} f & \left(\frac{a z+b}{c z+d}\right) \\
& =f_{0}(z)+f_{1}(z)\left(\frac{c}{c z+d}\right)+\cdots+f_{m}(z)\left(\frac{c}{c z+d}\right)^{m}
\end{aligned}
$$

for all $z \in \mathcal{H}$ and $\left(\begin{array}{ll}a & b \\ c & d\end{array}\right) \in \Gamma$, and the corresponding quasimodular polynomial has the functions $f_{k}$ as its coefficients.

In this paper we consider a particular automorphism of the space of such polynomials and prove that it is $\mathrm{SL}(2, \mathbb{R})$-equivariant with respect to the above-mentioned actions. The resulting isomorphism between the space of quasimodular polynomials and that of modular series determines a correspondence between quasimodular forms and some finite sequences of classical modular forms. More specifically, if a quasimodular form $f$ satisfies (1.1), the corresponding holomorphic functions $f_{k}$ can be expressed as linear combinations of derivatives of some modular forms. Furthermore, such modular forms can be written as linear combinations of derivatives of the functions $f_{k}$. We also use the equivariant automorphism to construct Poincaré series for quasimodular forms.

2. Correspondences of polynomials. In this section we consider polynomials whose coefficients are functions on the upper half-plane. We construct a linear automorphism of the space of such polynomials associated to each integer, which will be used in Section 4 to obtain modular forms corresponding to quasimodular forms.

Let $\mathcal{H}$ be the Poincaré upper half-plane, and let $\mathcal{F}$ be the ring of holomorphic functions $f: \mathcal{H} \rightarrow \mathbb{C}$ satisfying the growth condition

$$
|f(z)| \ll\left(\frac{\Im z}{1+|z|^{2}}\right)^{-\nu}
$$

for some $\nu>0$ (see e.g. [8, Section 17.1] for a more precise description of this condition). We fix a nonnegative integer $m$ and denote by $\mathcal{F}_{m}[X]$ the complex vector space of polynomials in $X$ over $\mathcal{F}$ of degree at most $m$. If an element $\Phi(z, X) \in \mathcal{F}_{m}[X]$ is a polynomial of the form

$$
\Phi(z, X)=\sum_{r=0}^{m} \phi_{r}(z) X^{r}
$$

and if $\lambda$ is an integer with $\lambda \geq 2 m$, we consider two other polynomials

$$
\left(\Xi_{\lambda}^{m} \Phi\right)(z, X),\left(\Lambda_{\lambda}^{m} \Phi\right)(z, X) \in \mathcal{F}_{m}[X]
$$


defined by

$$
\left(\Xi_{\lambda}^{m} \Phi\right)(z, X)=\sum_{r=0}^{m} \phi_{r}^{\Xi}(z) X^{r}, \quad\left(\Lambda_{\lambda}^{m} \Phi\right)(z, X)=\sum_{r=0}^{m} \phi_{r}^{\Lambda}(z) X^{r}
$$

where

$$
\begin{aligned}
\phi_{k}^{\Xi}= & \frac{1}{k !} \sum_{r=0}^{m-k} \frac{1}{r !(\lambda-2 k-r-1) !} \phi_{m-k-r}^{(r)}, \\
\phi_{k}^{\Lambda}= & (\lambda+2 k-2 m-1) \sum_{r=0}^{k} \frac{(-1)^{r}}{r !}(m-k+r) ! \\
& \times(2 k+\lambda-2 m-r-2) ! \phi_{m-k+r}^{(r)}
\end{aligned}
$$

for each $k \in\{0,1, \ldots, m\}$.

Lemma 2.1. Let $u$ and $v$ be positive integers with $u \leq v$. Then

$$
\sum_{r=0}^{u}(-1)^{r}\left(\begin{array}{l}
u \\
r
\end{array}\right)\left(\begin{array}{l}
v-r \\
u-1
\end{array}\right)=0 .
$$

Proof. See e.g. [6, Lemma 2.7].

Proposition 2.2. The maps $\Xi_{\lambda}^{m}, \Lambda_{\lambda}^{m}: \mathcal{F}_{m}[X] \rightarrow \mathcal{F}_{m}[X]$ given by $(2.3)$ are complex linear isomorphisms with

$$
\left(\Lambda_{\lambda}^{m}\right)^{-1}=\Xi_{\lambda}^{m} \quad \text { for each } \lambda>2 m .
$$

Proof. Given $\lambda>2 m$, we first consider a polynomial $\Phi(z, X)$ and its image $\left(\Xi_{\lambda}^{m} \Phi\right)(z, X)$ under $\Xi_{\lambda}$ as in (2.2) and (2.3), respectively. Then from (2.4), we obtain

$$
\phi_{m-k+r}^{\Xi}=\frac{1}{(m-k+r) !} \sum_{l=0}^{k-r} \frac{1}{l !(\lambda-2 m+2 k-2 r-l-1) !} \phi_{k-l-r}^{(l)}
$$

for $0 \leq r \leq k \leq m$. Thus, if we set

$$
\left(\left(\Lambda_{\lambda}^{m} \circ \Xi_{\lambda}^{m}\right) \Phi\right)(z, X)=\sum_{r=0}^{\infty} \widehat{\phi}_{r}(z) X^{r}
$$

then from (2.5) we see that

$$
\begin{aligned}
\frac{\widehat{\phi}_{k}}{\lambda+} & 2 k-2 m-1 \\
= & \sum_{r=0}^{k} \frac{(-1)^{r}}{r !}(m-k+r) !(\lambda+2 k-2 m-r-2) !\left(\phi_{m-k+r}^{\Xi}\right)^{(r)} \\
= & \sum_{r=0}^{k} \sum_{l=0}^{k-r} \frac{(-1)^{r}(\lambda+2 k-2 m-r-2) !}{r ! l !(\lambda-2 m+2 k-2 r-l-1) !} \phi_{k-l-r}^{(l+r)}
\end{aligned}
$$




$$
\begin{aligned}
= & \sum_{u=0}^{k} \sum_{r=0}^{u} \frac{(-1)^{r}(\lambda+2 k-2 m-r-2) !}{r !(u-r) !(\lambda+2 k-2 m-r-u-1) !} \phi_{k-u}^{(u)} \\
= & \frac{(\lambda+2 k-2 m-2) !}{(\lambda+2 k-2 m-1) !} \phi_{k} \\
& +\sum_{u=1}^{k} \frac{1}{u} \phi_{k-u}^{(u)} \sum_{r=0}^{u} \frac{(-1)^{r} u !(\lambda+2 k-2 m-r-2) !}{r !(u-r) !(\lambda+2 k-2 m-r-u-1) !(u-1) !} \\
= & \frac{\phi_{k}}{\lambda+2 k-2 m-1}+\sum_{u=1}^{k} \frac{1}{u} \phi_{k-u}^{(u)} \sum_{r=0}^{u}(-1)^{r}\left(\begin{array}{c}
u \\
r
\end{array}\right)\left(\begin{array}{c}
\lambda+2 k-2 m-r-2 \\
u-1
\end{array}\right) .
\end{aligned}
$$

Since $\lambda>2 m$, if $1 \leq u \leq k$, we have

$$
\lambda+2 k-2 m-2 \geq 2 k-1 \geq k \geq u \text {. }
$$

Thus, using Lemma 2.1, we see that

$$
\sum_{r=0}^{u}(-1)^{r}\left(\begin{array}{l}
u \\
r
\end{array}\right)\left(\begin{array}{c}
\lambda+2 k-2 m-r-2 \\
u-1
\end{array}\right)=0
$$

for $1 \leq u \leq k$. Hence (2.6) can be written in the form

$$
\frac{\widehat{\phi}_{k}}{\lambda+2 k-2 m-1}=\frac{\phi_{k}}{\lambda+2 k-2 m-1},
$$

and we obtain

$$
\left(\left(\Lambda_{\lambda}^{m} \circ \Xi_{\lambda}^{m}\right) \Phi\right)(z, X)=\Phi(z, X) .
$$

We now assume that $\left(\Lambda_{\lambda}^{m} \Phi\right)(z, X)$ is as in (2.3) and that

$$
\left(\left(\Xi_{\lambda}^{m} \circ \Lambda_{\lambda}^{m}\right) \Phi\right)(z, X)=\sum_{r=0}^{m} \widetilde{\phi}_{r}(z) X^{r} .
$$

Thus, in particular, $(2.5)$ is valid for $0 \leq k \leq m$. We shall verify that $\widetilde{\phi}_{k}=\phi_{k}$ for $0 \leq k \leq m$ using induction. Given a nonnegative integer $n<m$, we first assume that

$$
\phi_{k}=\widetilde{\phi}_{k}=\frac{1}{k !} \sum_{r=0}^{m} \frac{1}{r !(\lambda-2 k-r-1) !}\left(\phi_{m-k-r}^{\Lambda}\right)^{(r)}
$$

for each $k \in\{m-n, \ldots, m\}$. Then from (2.5) we obtain

$$
\begin{aligned}
\phi_{n+1}^{\Lambda}= & (2 n-\lambda-2 m+1) \\
& \times \sum_{r=0}^{n+1} \frac{(-1)^{r}}{r !}(2 n+\lambda-2 m-r) !(m-n-1+r) ! \phi_{m-n-1+r}^{(r)}
\end{aligned}
$$




$$
\begin{gathered}
=(2 n-\lambda-2 m+1) !(m-n-1) ! \phi_{m-n-1}-(2 n-\lambda-2 m+1) \\
\times \sum_{r=0}^{n} \frac{(-1)^{r}}{(r+1) !}(2 n+\lambda-2 m-r-1) !(m-n+r) ! \phi_{m-n+r}^{(r+1)},
\end{gathered}
$$

which can be written in the form

$$
\begin{aligned}
\phi_{m-n-1}= & \frac{\phi_{n+1}^{\Lambda}}{(2 n-\lambda-2 m+1) !(m-n-1) !} \\
& +\frac{1}{(2 n-\lambda-2 m) !(m-n-1) !} \\
& \times \sum_{r=0}^{n} \frac{(-1)^{r}}{(r+1) !}(2 n+\lambda-2 m-r-1) !(m-n+r) ! \phi_{m-n+r}^{(r+1)} .
\end{aligned}
$$

However, since (2.4) holds for $k \in\{m-n, \ldots, m\}$, we have

$$
\begin{aligned}
& \sum_{r=0}^{n} \frac{(-1)^{r}}{(r+1) !}(2 n+\lambda-2 m-r-1) !(m-n+r) ! \phi_{m-n+r}^{(r+1)} \\
& \quad=\sum_{r=0}^{n} \sum_{l=0}^{n-r} \frac{(-1)^{r}(2 n+\lambda-2 m-r-1) !}{(r+1) ! l !(\lambda-2 m+2 n-2 r-l-1) !}\left(\phi_{n-l-r}^{\Lambda}\right)^{(l+r+1)} \\
& \quad=\sum_{w=0}^{n} \sum_{r=0}^{w} \frac{(-1)^{r}(2 n+\lambda-2 m-r-1) !}{(r+1) !(w-r) !(\lambda-2 m+2 n-r-w-1) !}\left(\phi_{n-w}^{\Lambda}\right)^{(w+1)} \\
& =\sum_{w=0}^{n} \frac{1}{w+1} \sum_{r=0}^{w}(-1)^{r}\left(\begin{array}{c}
w+1 \\
r+1
\end{array}\right)\left(\begin{array}{c}
2 n+\lambda-2 m-r-1 \\
w
\end{array}\right)\left(\phi_{n-w}^{\Lambda}\right)^{(w+1)} .
\end{aligned}
$$

On the other hand, using Lemma 2.1, we deduce that

$$
\sum_{r=0}^{w}(-1)^{r}\left(\begin{array}{c}
w+1 \\
r+1
\end{array}\right)\left(\begin{array}{c}
2 n+\lambda-2 m-r-1 \\
w
\end{array}\right)=\left(\begin{array}{c}
2 n+\lambda-2 m \\
w
\end{array}\right) .
$$

Thus (2.7) can now be written as

$$
\begin{aligned}
\phi_{m-n-1}= & \frac{\phi_{n+1}^{\Lambda}}{(2 n-\lambda-2 m+1) !(m-n-1) !}+\frac{1}{(2 n-\lambda-2 m) !(m-n-1) !} \\
& \times \sum_{w=0}^{n} \frac{1}{w+1}\left(\begin{array}{c}
2 n+\lambda-2 m \\
w
\end{array}\right)\left(\phi_{n-w}^{\Lambda}\right)^{(w+1)} \\
= & \frac{\phi_{n+1}^{\Lambda}}{(2 n-\lambda-2 m+1) !(m-n-1) !}
\end{aligned}
$$




$$
\begin{gathered}
+\frac{1}{(2 n-\lambda-2 m) !(m-n-1) !} \sum_{w=1}^{n+1} \frac{1}{w}\left(\begin{array}{c}
2 n+\lambda-2 m \\
w-1
\end{array}\right)\left(\phi_{n+1-w}^{\Lambda}\right)^{(w)} \\
=\frac{1}{(m-n-1) !} \sum_{w=0}^{n+1} \frac{1}{w !(2 n+\lambda-2 m-w+1) !}\left(\phi_{n+1-w}^{\Lambda}\right)^{(w)}=\widetilde{\phi}_{m-n-1} .
\end{gathered}
$$

Hence $\widetilde{\phi}_{k}=\phi_{k}$ for all $n \in\{0,1, \ldots, m\}$ by induction. Thus

$$
\left(\left(\Xi_{\lambda}^{m} \circ \Lambda_{\lambda}^{m}\right) \Phi\right)(z, X)=\Phi(z, X),
$$

and the proof of the proposition is complete.

3. Modular and quasimodular polynomials. In this section we introduce two types of actions of $\mathrm{SL}(2, \mathbb{R})$ on the space of polynomials considered in Section 2. We show that the isomorphisms in Proposition 2.2 are equivariant with respect to these actions. As an application, we establish a correspondence between modular and quasimodular polynomials.

The group $\operatorname{SL}(2, \mathbb{R})$ acts on the Poincaré upper half-plane $\mathcal{H}$ as usual by linear fractional transformations, so that

$$
\gamma z=\frac{a z+b}{c z+d}
$$

for all $z \in \mathcal{H}$ and $\gamma=\left(\begin{array}{ll}a & b \\ c & d\end{array}\right) \in \operatorname{SL}(2, \mathbb{R})$. For the same $z$ and $\gamma$, we set

$$
\mathfrak{J}(\gamma, z)=c z+d, \quad \mathfrak{K}(\gamma, z)=\frac{c}{c z+d} .
$$

These formulas determine the maps $\mathfrak{J}, \mathfrak{K}: \mathrm{SL}(2, \mathbb{R}) \times \mathcal{H} \rightarrow \mathbb{C}$ of which the first map is the usual automorphy factor satisfying the cocycle condition

$$
\mathfrak{J}\left(\gamma \gamma^{\prime}, z\right)=\mathfrak{J}\left(\gamma, \gamma^{\prime} z\right) \mathfrak{J}\left(\gamma^{\prime}, z\right)
$$

for all $\gamma, \gamma^{\prime} \in \mathrm{SL}(2, \mathbb{R})$ and $z \in \mathcal{H}$. On the other hand, it can be shown that the second map satisfies

$$
\mathfrak{K}\left(\gamma \gamma^{\prime}, z\right)=\mathfrak{K}\left(\gamma^{\prime}, z\right)+\mathfrak{J}\left(\gamma^{\prime}, z\right)^{-2} \mathfrak{K}\left(\gamma, \gamma^{\prime} z\right) .
$$

Let $\mathcal{F}$ be the ring of holomorphic functions $f: \mathcal{H} \rightarrow \mathbb{C}$ satisfying (2.1), as in Section 2. If $\gamma \in \mathrm{SL}(2, \mathbb{R}), \lambda \in \mathbb{Z}, f \in \mathcal{F}$ and

$$
\Phi(z, X)=\sum_{r=0}^{m} \phi_{r}(z) X^{r} \in \mathcal{F}_{m}[X],
$$

we set

$$
\left(\left.f\right|_{\lambda} \gamma\right)(z)=\mathfrak{J}(\gamma, z)^{-\lambda} f(\gamma z)
$$




$$
\begin{gathered}
\left(\left.\Phi\right|_{\lambda} ^{X} \gamma\right)(z, X)=\sum_{r=0}^{m}\left(\left.\phi_{r}\right|_{\lambda+2 r} \gamma\right)(z) X^{r}, \\
\left(\Phi \|_{\lambda} \gamma\right)(z, X)=\mathfrak{J}(\gamma, z)^{-\lambda} \Phi\left(\gamma z, \mathfrak{J}(\gamma, z)^{2}(X-\mathfrak{K}(\gamma, z))\right)
\end{gathered}
$$

for all $z \in \mathcal{H}$. If $\gamma^{\prime}$ is another element of $\operatorname{SL}(2, \mathbb{R})$, then it can be shown that

$$
\begin{aligned}
\left(\left.f\right|_{\lambda}\left(\gamma \gamma^{\prime}\right)\right)(z) & =\left(\left.\left(\left.f\right|_{\lambda} \gamma\right)\right|_{\lambda} \gamma^{\prime}\right)(z), \\
\left(\left.\Phi\right|_{\lambda} ^{X}\left(\gamma \gamma^{\prime}\right)\right)(z, X) & =\left(\left(\left.\Phi\right|_{\lambda} ^{X} \gamma\right) \|_{\lambda} \gamma^{\prime}\right)(z, X), \\
\left(\Phi \|_{\lambda}\left(\gamma \gamma^{\prime}\right)\right)(z, X) & =\left(\left(\Phi \|_{\lambda} \gamma\right) \|_{\lambda} \gamma^{\prime}\right)(z, X) .
\end{aligned}
$$

Thus the above operations determine right actions of $\operatorname{SL}(2, \mathbb{R})$, one action on $\mathcal{F}$ and the other two on $\mathcal{F}_{m}[X]$. We note that

$$
\left(\left.f\right|_{\lambda} \gamma\right)^{(r)}(z)=\sum_{l=0}^{r}(-1)^{r-l} \frac{r !}{l !}\left(\begin{array}{c}
\lambda+r-1 \\
r-l
\end{array}\right) \frac{\mathfrak{K}(\gamma, z)^{r-l}}{\mathfrak{J}(\gamma, z)^{\lambda+2 l}} f^{(l)}(\gamma z)
$$

for $f \in \mathcal{F}, z \in \mathcal{H}, \gamma \in \mathrm{SL}(2, \mathbb{R})$ and $r \geq 0$ (cf. [3, (1.9)]).

TheOREM 3.1. Given a polynomial $\Phi(z, X) \in \mathcal{F}_{m}[X]$ and an integer $\lambda$, we have

$$
\begin{aligned}
\left(\left(\Xi_{\lambda}^{m} \Phi\right) \|_{\lambda} \gamma\right)(z, X) & =\Xi_{\lambda}^{m}\left(\left.\Phi\right|_{\lambda-2 m} ^{X} \gamma\right)(z, X), \\
\left(\left.\left(\Lambda_{\lambda}^{m} \Phi\right)\right|_{\lambda-2 m} ^{X} \gamma\right)(z, X) & =\Lambda_{\lambda}^{m}\left(\Phi \|_{\lambda} \gamma\right)(z, X)
\end{aligned}
$$

for all $\gamma \in \mathrm{SL}(2, \mathbb{R})$, where $\Xi_{\lambda}^{m}$ and $\Lambda_{\lambda}^{m}$ are the isomorphisms in Proposition 2.2.

Proof. Let $\Phi(z, X) \in \mathcal{F}_{m}[X]$ be given by (3.4), so that

$$
\left(\Xi_{\lambda}^{m} \Phi\right)(z, X)=\sum_{r=0}^{m} \phi_{r}^{\Xi}(z) X^{r}
$$

for $\lambda \in \mathbb{Z}$, where the coefficients $\phi_{r}^{\Xi}(z)$ are as in $(2.4)$. If $\gamma \in \operatorname{SL}(2, \mathbb{R})$, from (3.7) we obtain

$$
\begin{aligned}
\left(\left(\Xi_{\lambda}^{m} \Phi\right) \|_{\lambda} \gamma\right)(z, X) & =\mathfrak{J}(\gamma, z)^{-\lambda} \sum_{l=0}^{m} \phi_{l}^{\Xi}(\gamma z) \mathfrak{J}(\gamma, z)^{2 l}(X-\mathfrak{K}(\gamma, z))^{l} \\
& =\sum_{l=0}^{m} \sum_{r=0}^{l}\left(\begin{array}{l}
l \\
r
\end{array}\right) \phi_{l}^{\Xi}(\gamma z) \mathfrak{J}(\gamma, z)^{2 l-\lambda}(-1)^{l-r} \mathfrak{K}(\gamma, z)^{l-r} X^{r} \\
& =\sum_{r=0}^{m} \sum_{l=r}^{m}(-1)^{l-r}\left(\begin{array}{l}
l \\
r
\end{array}\right) \phi_{l}^{\Xi}(\gamma z) \mathfrak{J}(\gamma, z)^{2 l-\lambda} \mathfrak{K}(\gamma, z)^{l-r} X^{r} .
\end{aligned}
$$

Thus we may write

$$
\left(\left(\Xi_{\lambda}^{m} \Phi\right) \|_{\lambda} \gamma\right)(z, X)=\sum_{r=0}^{m} \xi_{r}^{\Xi}(\gamma, z) X^{r},
$$


where

$$
\xi_{r}^{\Xi}(\gamma, z)=\sum_{l=0}^{m-r}(-1)^{l}\left(\begin{array}{c}
l+r \\
r
\end{array}\right) \phi_{l+r}^{\Xi}(\gamma z) \mathfrak{J}(\gamma, z)^{2 l+2 r-\lambda} \mathfrak{K}(\gamma, z)^{l}
$$

for each $r \geq 0$. Using (2.4), we have

$$
\phi_{l+r}^{\Xi}(\gamma z)=\frac{1}{(l+r) !} \sum_{k=0}^{m-r-l} \frac{1}{k !(\lambda-2 r-2 l-k-1) !} \phi_{m-r-l-k}^{(k)}(\gamma z),
$$

and therefore we obtain

$$
\xi_{r}^{\Xi}(\gamma, z)=\sum_{l=0}^{m-r} \sum_{k=0}^{m-r-l} \frac{(-1)^{l} \mathfrak{J}(\gamma, z)^{2 l+2 r-\lambda} \mathfrak{K}(\gamma, z)^{l}}{k ! r ! l !(\lambda-2 r-2 l-k-1) !} \phi_{m-r-l-k}^{(k)}(\gamma z) .
$$

On the other hand, from (2.3), (2.4) and (3.6) we see that

$$
\Xi_{\lambda}^{m}\left(\left.\Phi\right|_{\lambda-2 m} ^{X} \gamma\right)(z, X)=\sum_{r=0}^{m} \eta_{r}^{\Xi}(\gamma, z) X^{r},
$$

where

$$
\eta_{r}^{\Xi}(\gamma, z)=\frac{1}{r !} \sum_{k=0}^{m-r} \frac{1}{k !(\lambda-2 r-k-1) !}\left(\left.\phi_{m-r-k}\right|_{\lambda-2 r-2 k} \gamma\right)^{(k)}(z)
$$

for $r \geq 0$. Using (3.8), we have

$$
\begin{aligned}
& \left(\left.\phi_{m-r-k}\right|_{\lambda-2 r-2 k} \gamma\right)^{(k)}(z) \\
& \quad=\sum_{l=0}^{k}(-1)^{k-l} \frac{k !}{l !}\left(\begin{array}{c}
\lambda-2 r-k-1 \\
k-l
\end{array}\right) \frac{\mathfrak{K}(\gamma, z)^{k-l}}{\mathfrak{J}(\gamma, z)^{\lambda-2 r-2 k+2 l}} \phi_{m-r-k}^{(l)}(\gamma z) .
\end{aligned}
$$

Thus we obtain

$$
\begin{aligned}
\eta_{r}(\gamma, z) & =\sum_{k=0}^{m-r} \sum_{l=0}^{k} \frac{(-1)^{k-l} \mathfrak{K}(\gamma, z)^{k-l} \mathfrak{J}(\gamma, z)^{-\lambda+2 r+2 k-2 l}}{r ! l !(k-l) !(\lambda-2 r-2 k+l-1) !} \phi_{m-r-k}^{(l)}(\gamma z) \\
& =\sum_{l=0}^{m-r} \sum_{k=l}^{m-r} \frac{(-1)^{k-l} \mathfrak{K}(\gamma, z)^{k-l} \mathfrak{J}(\gamma, z)^{-\lambda+2 r+2 k-2 l}}{r ! l !(k-l) !(\lambda-2 r-2 k+l-1) !} \phi_{m-r-k}^{(l)}(\gamma z) \\
& =\sum_{l=0}^{m-r} \sum_{k=0}^{m-l-r} \frac{(-1)^{k} \mathfrak{K}(\gamma, z)^{k} \mathfrak{J}(\gamma, z)^{-\lambda+2 r+2 k}}{r ! l ! k !(\lambda-2 r-2 k-l-1) !} \phi_{m-r-k-l}^{(l)}(\gamma z) .
\end{aligned}
$$

Comparing this with (3.11), we have

$$
\xi_{r}(\gamma, z)=\eta_{r}(\gamma, z)
$$

for each $r \in\{0, \ldots, m\}$, which verifies (3.9). The relation (3.10) follows from this and Proposition 2.2. 
Definition 3.2. Let $\Gamma$ be a discrete subgroup of $\operatorname{SL}(2, \mathbb{R})$, and let $\left.\right|_{\lambda}$, $\left.\right|_{\lambda} ^{X}$ and $\|_{\lambda}$ with $\lambda \in \mathbb{Z}$ be the operations in (3.5)-(3.7).

(i) An element $f \in \mathcal{F}$ is a modular form for $\Gamma$ of weight $\lambda$ if it satisfies

$$
\left.f\right|_{\lambda} \gamma=f
$$

for all $\gamma \in \Gamma$. We denote by $M_{\lambda}(\Gamma)$ the space of modular forms for $\Gamma$ of weight $\lambda$.

(ii) An element $F(z, X) \in \mathcal{F}_{m}[X]$ is a modular polynomial for $\Gamma$ of weight $\lambda$ and degree at most $m$ if it satisfies

$$
\left.F\right|_{\lambda} ^{X} \gamma=F
$$

for all $\gamma \in \Gamma$. We use $M P_{\lambda}^{m}(\Gamma)$ to denote the space of modular polynomials for $\Gamma$ of weight $\lambda$ and degree at most $m$.

(iii) An element $\Phi(z, X) \in \mathcal{F}_{m}[X]$ is a quasimodular polynomial for $\Gamma$ of weight $\lambda$ and degree at most $m$ if it satisfies

$$
\Phi \|_{\lambda} \gamma=\Phi
$$

for all $\gamma \in \Gamma$. We denote by $Q P_{\lambda}^{m}(\Gamma)$ the space of quasimodular polynomials for $\Gamma$ of weight $\lambda$ and degree at most $m$.

If a polynomial $F(z, X) \in \mathcal{F}_{m}[X]$ of the form

$$
F(z, X)=\sum_{r=0}^{m} f_{r}(z) X^{r}
$$

belongs to $M P_{\lambda}^{m}(\Gamma)$, from (3.6) and Definition 3.2(ii) we see that

$$
f_{r} \in M_{\lambda+2 r}(\Gamma)
$$

for $0 \leq r \leq m$.

Proposition 3.3. The isomorphisms $\Xi_{\lambda}^{m}$ and $\Lambda_{\lambda}^{m}$ in Proposition 2.2 induce the isomorphisms

$$
\Xi_{\lambda}^{m}: M P_{\lambda-2 m}^{m}(\Gamma) \rightarrow Q P_{\lambda}^{m}(\Gamma), \quad \Lambda_{\lambda}^{m}: Q P_{\lambda}^{m}(\Gamma) \rightarrow M P_{\lambda-2 m}^{m}(\Gamma)
$$

for each $\lambda \in \mathbb{Z}$.

Proof. This follows immediately from Theorem 3.1 and Definition 3.2.

4. Quasimodular forms. In this section we study some of the properties of quasimodular forms including their correspondence with finite sequences of modular forms. We also construct Poincaré series for quasimodular forms.

Let $\Gamma$ be a discrete subgroup of $\operatorname{SL}(2, \mathbb{R})$, and let $\mathcal{F}$ be as in Section 2 . Given a nonnegative integer $m$, let $\mathcal{F}_{m}[X]$ be as in Section 3 . 
Definition 4.1. Given an integer $\lambda$, an element $f \in \mathcal{F}$ is a quasimodular form for $\Gamma$ of weight $\lambda$ and depth at most $m$ if there are functions $f_{0}, \ldots, f_{m} \in \mathcal{F}$ such that

$$
\left(\left.f\right|_{\lambda} \gamma\right)(z)=\sum_{r=0}^{m} f_{r}(z) \mathfrak{K}(\gamma, z)^{r}
$$

for all $z \in \mathcal{H}$ and $\gamma \in \Gamma$, where $\mathfrak{K}(\gamma, z)$ is as in (3.1) and $\left.\right|_{\lambda}$ is the operation in (3.5). We denote by $Q M_{\lambda}^{m}(\Gamma)$ the space of quasimodular forms for $\Gamma$ of weight $\lambda$ and depth at most $m$.

REMARK 4.2.

(i) If $\gamma \in \Gamma$ is the identity matrix in (4.1), then $\mathfrak{K}(\gamma, z)=0$, and therefore it follows that

$$
f=f_{0} .
$$

On the other hand, if $m=0$, the relation (4.1) can be written in the form

$$
\left.f\right|_{\lambda} \gamma=f_{0}=f
$$

hence we see that $Q M_{\lambda}^{0}(\Gamma)$ coincides with the space $M_{\lambda}(\Gamma)$ of modular forms.

(ii) If (4.1) is satisfied for another set of functions $\widehat{f}_{0}, \ldots, \widehat{f}_{m} \in \mathcal{F}$, then we have

$$
\sum_{r=0}^{m}\left(\widehat{f}_{r}(z)-f_{r}(z)\right) \mathfrak{K}(\gamma, z)^{r}=0
$$

for all $\gamma$ belonging to the infinite set $\Gamma$; hence it follows that $\widehat{f}_{r}=f_{r}$ for each $r$. Thus we see that the quasimodular form $f$ determines the associated functions $f_{0}, \ldots, f_{m} \in \mathcal{F}$ uniquely.

Let $f \in \mathcal{F}$ be a quasimodular form belonging to $Q M_{\lambda}^{m}(\Gamma)$ satisfying (4.1). Then we define the corresponding polynomial $\left(\mathcal{Q}_{\lambda}^{m} f\right)(z, X) \in \mathcal{F}_{m}[X]$ by

$$
\left(\mathcal{Q}_{\lambda}^{m} f\right)(z, X)=\sum_{r=0}^{m} f_{r}(z) X^{r}
$$

for all $z \in \mathcal{H}$. From Remark 4.2(ii) we see that $\mathcal{Q}_{\lambda}^{m} f$ is well-defined; hence we obtain the complex linear map

$$
\mathcal{Q}_{\lambda}^{m}: Q M_{\lambda}^{m}(\Gamma) \rightarrow \mathcal{F}_{m}[X]
$$

for each $\lambda \in \mathbb{Z}$.

Lemma 4.3. Let $F(z, X)=\sum_{r=0}^{m} f_{r}(z) X^{r} \in \mathcal{F}_{m}[X]$. Then $F(z, X)$ is a quasimodular polynomial belonging to $Q P_{\lambda}^{m}(\Gamma)$ if and only if for each 
$r \in\{0,1, \ldots, m\}$ the coefficient $f_{r}$ satisfies

$$
\left(\left.f_{r}\right|_{\lambda-2 r} \gamma\right)(z)=\sum_{l=0}^{m-r}\left(\begin{array}{c}
l+r \\
r
\end{array}\right) f_{l+r}(z) \mathfrak{K}(\gamma, z)^{l}
$$

for all $z \in \mathcal{H}$ and $\gamma \in \Gamma$. In particular, $f_{r}$ is a quasimodular form belonging to $Q M_{\lambda-2 r}^{m-r}(\Gamma)$.

Proof. See e.g. [2].

If $0 \leq l \leq m$, we consider the complex linear map

$$
\mathfrak{S}_{l}: \mathcal{F}_{m}[X] \rightarrow \mathcal{F} \quad \text { defined by } \quad \mathfrak{S}_{l}\left(\sum_{r=0}^{m} f_{r}(z) X^{r}\right)=f_{l}(z)
$$

for all $z \in \mathcal{H}$. Then from Lemma 4.3 we see that

$$
\mathfrak{S}_{l}\left(Q P_{\lambda}^{m}(\Gamma)\right) \subset Q M_{\lambda-2 l}^{m-l}(\Gamma) ;
$$

hence we obtain the map

$$
\mathfrak{S}_{l}: Q P_{\lambda}^{m}(\Gamma) \rightarrow Q M_{\lambda-2 l}^{m-l}(\Gamma)
$$

for each $l$. On the other hand, using (4.2) and (4.3), we also have

$$
\left(\mathcal{Q}_{\lambda-2 r}^{m-r}\left(\mathfrak{S}_{r} F\right)\right)(z, X)=\sum_{l=0}^{m-r}\left(\begin{array}{c}
l+r \\
r
\end{array}\right)\left(\mathfrak{S}_{l+r} F\right)(z) X^{l} \in Q P_{\lambda-2 r}^{m-r}(\Gamma)
$$

for $F(z, X) \in Q M_{\lambda}^{m}(\Gamma)$ and $0 \leq r \leq m$. In particular, the map $\mathcal{Q}_{\lambda}^{m}$ given by (4.2) determines the complex linear map

$$
\mathcal{Q}_{\lambda}^{m}: Q M_{\lambda}^{m}(\Gamma) \rightarrow Q P_{\lambda}^{m}(\Gamma)
$$

for each $\lambda \in \mathbb{Z}$.

Lemma 4.4. The map $\mathfrak{S}_{0}: Q P_{\lambda}^{m}(\Gamma) \rightarrow Q M_{\lambda}^{m}(\Gamma)$ in (4.4) is an isomorphism whose inverse is the map $\mathcal{Q}_{\lambda}^{m}$ in (4.5).

Proof. See [2].

Let $\phi \in Q M_{\lambda}^{m}(\Gamma)$ be a quasimodular form satisfying

$$
\left(\left.\phi\right|_{\lambda} \gamma\right)(z)=\sum_{r=0}^{m} \phi_{r}(z) \mathfrak{K}(\gamma, z)^{r}
$$

for all $\gamma \in \Gamma$ and $z \in \mathcal{H}$, so that

$$
\left(\mathcal{Q}_{\lambda}^{m} \phi\right)(z, X)=\sum_{r=0}^{m} \phi_{r}(z) X^{r} \in Q P_{\lambda}^{m}(\Gamma)
$$

by (4.2). We then set

$$
\widetilde{\Lambda}_{\lambda}^{m}(\phi)=\left(\phi_{0}^{\Lambda}, \phi_{1}^{\Lambda}, \ldots, \phi_{m}^{\Lambda}\right) \in \mathcal{F}^{m+1},
$$


where the functions $\phi_{r}^{\Lambda} \in \mathcal{F}$ are the coefficients of the modular polynomial

$$
\left(\left(\Lambda_{\lambda}^{m} \circ \mathcal{Q}_{\lambda}^{m}\right) \phi\right)(z, X) \in M P_{\lambda}^{m}(\Gamma)
$$

given by (2.5).

Proposition 4.5. The formula (4.7) determines an isomorphism

$$
\widetilde{\Lambda}_{\lambda}^{m}: Q M_{\lambda}^{m}(\Gamma) \rightarrow \bigoplus_{k=0}^{m} M_{\lambda+2 k-2 m}(\Gamma)
$$

of complex vector spaces for $\lambda>2 m$.

Proof. From (3.12) we see that $\phi_{r}^{\Lambda} \in M_{\lambda+2 r}(\Gamma)$ for each $r \in\{0,1, \ldots, m\}$; hence the proposition follows from Lemma 4.4.

EXAMPLE 4.6. We consider modular forms $g \in M_{2}(\Gamma)$ and $p \in M_{\xi}(\Gamma)$ with $\xi \in \mathbb{Z}$. Then we see that $g^{k} p \in M_{\xi+2 k}(\Gamma)$ for $0 \leq k \leq m$; hence

$$
\left(p, g p, \ldots, g^{m} p\right) \in \bigoplus_{k=0}^{m} M_{\lambda+2 k-2 m}(\Gamma) .
$$

Thus we obtain

$$
\begin{aligned}
\left(\widetilde{\Lambda}_{\xi+2 m}^{m}\right)^{-1}\left(p, g p, \ldots, g^{m} p\right) & =\sum_{r=0}^{m} \frac{1}{r !(\lambda-r-1) !}\left(g^{m-r} p\right)^{(r)} \\
& =\sum_{r=0}^{m} \sum_{l=0}^{r} \frac{1}{r !(\lambda-r-1) !}\left(\begin{array}{c}
r \\
l
\end{array}\right)\left(g^{m-r}\right)^{(l)} p^{(r-l)} \\
& =\sum_{r=0}^{m} \sum_{l=0}^{r} \frac{\left(g^{m-r}\right)^{(l)} p^{(r-l)}}{l !(r-l) !(\lambda-r-1) !}
\end{aligned}
$$

and it is a quasimodular form belonging to $Q M_{\xi+2 m}^{m}(\Gamma)$.

In order to discuss Poincaré series we now assume that $x$ is a cusp of $\Gamma$, so that there is an element $\sigma \in \operatorname{SL}(2, \mathbb{R})$ such that

$$
\sigma \Gamma_{x} \sigma^{-1} \cdot\{ \pm 1\}=\left\{ \pm\left(\begin{array}{cc}
1 & h \\
0 & 1
\end{array}\right)^{n} \mid n \in \mathbb{Z}\right\}
$$

for some positive real number $h$. Given integers $w \geq 3$ and $u \geq 0$, we set

$$
\mathcal{P}_{w, u}^{x}(z)=\sum_{\gamma \in \Gamma_{x} \backslash \Gamma} \mathfrak{J}(\sigma \gamma, z)^{-w} \mathbf{e}^{u / h}(\sigma \gamma z)=\left(\left.\mathbf{e}^{u / h}\right|_{2(\xi-m+r)} \sigma \gamma\right)(z)
$$

for all $z \in \mathcal{H}$, where $\mathbf{e}^{\mu}(\cdot)=\exp (2 \pi i \mu(\cdot))$ for $\mu \in \mathbb{C}$. Then it is well-known that the series in (4.9) converges absolutely and uniformly on any compact subset of $\mathcal{H}$, and the resulting function $\mathcal{P}_{w, u}^{x}: \mathcal{H} \rightarrow \mathbb{C}$ is a Poincaré series for modular forms belonging to $M_{w}(\Gamma)$. 
If $\alpha, u \in \mathbb{Z}$, we set

$$
\eta_{\alpha, u}(z)=\mathfrak{J}(\sigma, z)^{-\alpha} \mathbf{e}^{u / h}(\sigma z)
$$

for all $z \in \mathcal{H}$, where $h$ is as in (4.8). Then, using (3.2), we have

$$
\left(\left.\eta_{\alpha, u}\right|_{\alpha} \gamma\right)(z)=\mathfrak{J}(\gamma, z)^{-\alpha} \mathfrak{J}(\sigma, \gamma z)^{-\alpha} \mathbf{e}^{u / h}(\sigma \gamma z)=\mathfrak{J}(\sigma \gamma, z)^{-\alpha} \mathbf{e}^{u / h}(\sigma \gamma z) .
$$

Thus the Poincaré series (4.8) can be written in the form

$$
\mathcal{P}_{w, u}^{x}(z)=\sum_{\gamma \in \Gamma_{x} \backslash \Gamma}\left(\left.\eta_{\alpha, u}\right|_{\alpha} \gamma\right)(z) .
$$

Given $\xi \in \mathbb{Z}$, we consider the polynomial

$$
G_{\xi, u}(z, X)=\sum_{r=0}^{m} \eta_{2(\xi-m+r), u}(z) X^{r} \in \mathcal{F}_{m}[X],
$$

and set

$$
\widehat{\mathcal{P}}_{2 \xi, u}^{x}(z, X)=\sum_{\gamma \in \Gamma_{x} \backslash \Gamma}\left(\left(\Xi_{2 \xi}^{m} G_{\xi, u}\right) \|_{2 \xi-2 m} \gamma\right)(z, X)
$$

for $z \in \mathcal{H}$, where $x$ is a cusp of $\Gamma$ as above and $\Xi_{2 \xi}^{m}$ is as in (2.3).

THEOREM 4.7.

(i) The series $\widehat{\mathcal{P}}_{2 \xi, u}^{x}(z, X)$ given by (4.12) is a quasimodular polynomial belonging to $Q P_{2 \xi}^{m}(\Gamma)$.

(ii) The series $\widehat{\mathcal{P}}_{2 \xi, u}^{x}(z, X)$ can be written in the form

(4.13) $\quad \widehat{\mathcal{P}}_{2 \xi, u}^{x}(z, X)$

$$
\begin{aligned}
= & \sum_{\gamma \in \Gamma_{x} \backslash \Gamma} \sum_{r=0}^{m} \sum_{l=0}^{m-r} \sum_{j=0}^{l} \frac{(-1)^{l-j}(2 \pi i u)^{j} l !}{h^{j} j ! r !(2 \xi-2 m-l-1) !} \\
& \times\left(\begin{array}{c}
2 \xi-2 r-l-1 \\
l-j
\end{array}\right) \frac{\mathfrak{K}(\sigma \gamma, z)^{l-j}}{\mathfrak{J}(\sigma \gamma, z)^{2 \xi-2 r-2 l+2 j}} \mathbf{e}^{u / h}(\sigma \gamma z) X^{r} .
\end{aligned}
$$

(iii) The function $\widehat{\mathcal{P}}_{2 \xi, u}^{x, 0} \in \mathcal{F}$ given by

$$
\begin{aligned}
\widehat{\mathcal{P}}_{2 \xi, u}^{x, 0}(z)= & \sum_{\gamma \in \Gamma_{x} \backslash \Gamma} \sum_{l=0}^{m} \sum_{j=0}^{l} \frac{(-1)^{l-j}(2 \pi i u)^{j} l !}{h^{j} j !(2 \xi-2 m-l-1) !} \\
& \times\left(\begin{array}{c}
2 \xi-l-1 \\
l-j
\end{array}\right) \frac{\mathfrak{K}(\sigma \gamma, z)^{l-j}}{\mathfrak{J}(\sigma \gamma, z)^{2 \xi-2 r-2 l+2 j}} \mathbf{e}^{u / h}(\sigma \gamma z)
\end{aligned}
$$

for $z \in \mathcal{H}$ is a quasimodular form belonging to $Q M_{2 \xi}^{m}(\Gamma)$. 
Proof. Using (3.6), (4.10)-(4.12) and Theorem 3.1, we have

$$
\begin{aligned}
\widehat{\mathcal{P}}_{2 \xi, u}^{x}(z, X) & =\sum_{\gamma \in \Gamma_{x} \backslash \Gamma} \Xi_{2 \xi}^{m}\left(\left.G_{\xi, u}\right|_{2 \xi-2 m} ^{X} \gamma\right)(z, X) \\
& =\sum_{\gamma \in \Gamma_{x} \backslash \Gamma} \Xi_{2 \xi}^{m}\left(\sum_{r=0}^{m}\left(\left.\eta_{2(\xi-m+r), u}\right|_{2(\xi-m+r)} \gamma\right)(z) X^{r}\right) \\
& =\Xi_{2 \xi}^{m}\left(\sum_{r=0}^{m} \mathcal{P}_{2(\xi-m+r), u}(z) X^{r}\right) .
\end{aligned}
$$

Since $\mathcal{P}_{2(\xi-m+r), u} \in M_{2(\xi-m+r)}(\Gamma)$ for each $r \geq 0$, from (3.6) and Definition 3.2 we see that the sum

$$
F(z, X)=\sum_{r=0}^{m} \mathcal{P}_{2(\xi-m+r), u}(z) X^{r}
$$

is a modular polynomial belonging to $M P_{2 \xi-2 m}^{m}(\Gamma)$, and therefore by Proposition 3.3 its image

$$
\widehat{\mathcal{P}}_{2 \xi, u}^{x}(z, X)=\left(\Xi_{2 \xi}^{m} F\right)(z, X)
$$

under $\Xi_{2 \xi}^{m}$ is a quasimodular polynomial belonging to $Q P_{2 \xi}^{m}(\Gamma)$; hence (i) follows. On the other hand, using (2.3) (4.9), (4.14) and (4.15), we have

$$
\begin{aligned}
\left(\Xi_{2 \xi}^{m} F\right)(z, X) & =\sum_{\gamma \in \Gamma_{x} \backslash \Gamma} \Xi_{2 \xi}^{m}\left(\sum_{r=0}^{m}\left(\left.\mathbf{e}^{u / h}\right|_{2(\xi-m+r)} \sigma \gamma\right)(z) X^{r}\right) \\
& =\sum_{\gamma \in \Gamma_{x} \backslash \Gamma} \sum_{r=0}^{m} \widehat{\phi}_{r}(z) X^{r},
\end{aligned}
$$

where

$$
\widehat{\phi}_{r}=\frac{1}{r !} \sum_{l=0}^{m-r} \frac{1}{(2 \xi-2 r-l-1) !}\left(\left.\mathbf{e}^{u / h}\right|_{2(\xi-r-l)} \sigma \gamma\right)^{(l)} .
$$

However, from (3.8) we see that

$$
\begin{aligned}
& =\sum_{j=0}^{l}(-1)^{l-j} \frac{l !}{j !}\left(\begin{array}{c}
2 \xi-2 r-l-1 \\
l-j
\end{array}\right) \frac{\mathfrak{K}(\sigma \gamma, z)^{l-j}}{\mathfrak{J}(\sigma \gamma, z)^{2 \xi-2 r-2 l+2 j}}\left(\mathbf{e}^{u / h}\right)^{(j)}(\sigma \gamma z) \\
& =\sum_{j=0}^{l} \frac{(-1)^{l-j}(2 \pi i u)^{j} l !}{h^{j} j !}\left(\begin{array}{c}
2 \xi-2 r-l-1 \\
l-j
\end{array}\right) \frac{\mathfrak{K}(\sigma \gamma, z)^{l-j}}{\mathfrak{J}(\sigma \gamma, z)^{2 \xi-2 r-2 l+2 j}} \mathbf{e}^{u / h}(\sigma \gamma z) .
\end{aligned}
$$

Thus we obtain (4.15) by combining (4.16)-(4.18), which proves (ii). Finally, (iii) follows from (ii), Remark 4.2, and Lemma 4.4. 


\section{References}

[1] N. O. Azaiez, The ring of quasimodular forms for a cocompact group, preprint.

[2] Y. Choie and M. H. Lee, Quasimodular forms, Jacobi-like forms, and pseudodifferential operators, preprint.

[3] P. B. Cohen, Y. Manin and D. Zagier, Automorphic pseudodifferential operators, in: Algebraic Aspects of Nonlinear Systems, Birkhäuser, Boston, 1997, 17-47.

[4] A. Eskin and A. Okounkov, Asymptotics of numbers of branched coverings of a torus and volumes of moduli spaces of holomorphic differentials, Invent. Math. 145 (2001), 59-103.

[5] M. Kaneko and D. Zagier, A generalized Jacobi theta function and quasimodular forms, in: The Moduli Space of Curves, Progr. Math. 129, Birkhäuser, Boston, 1995, 165-172.

[6] M. H. Lee, Vector-valued Jacobi-like forms, Monatsh. Math. 152 (2007), 321-336.

[7] F. Martin and E. Royer, Rankin-Cohen brackets on quasimodular forms, math.NT/ 0509653.

[8] —, 一, Formes modulaires et périodes, in: Formes modulaires et transcendance, S. Fischler, É. Gaudron and S. Khémira (eds.), Sémin. Congr. 12, Soc. Math. France, Paris, 2005, 1-117.

[9] H. Movasati, On differential modular forms and some analytic relations between Eisenstein series, preprint.

[10] A. Okounkov and R. Pandharipande, Gromov-Witten theory, Hurwitz theory, and completed cycles, Ann. of Math. 163 (2006), 517-560.

[11] E. Royer, Evaluating convolution sums of the divisor function via quasimodular forms, Int. J. Number Theory 21 (2007), 231-262.

Department of Mathematics

University of Northern Iowa

Cedar Falls, IA 50614, U.S.A.

E-mail: lee@math.uni.edu

Received on 10.3.2008

and in revised form on 2.12.2008 